\title{
THE DEPARTMENT OF LABOUR'S JOB VACANCY MONITORING PROGRAMME
}

\author{
Andrew Whiteford \\ Labour Market Information Unit \\ Department of Labour
}

\begin{abstract}
This paper introduces the Department of Labour's Job Vacancy Monitoring Programme (JVMP). The programme is an initiative of the government's Skills Action Plan and will provide new insights into skill shortages. It was designed through a comprehensive process of stakeholder consultation and a review of the international experience with skill shortage and vacancy surveys. The paper provides an overview of this process as well as a short description of the components of JVMP. These components are:
\end{abstract}

- the Job Vacancy Monitor (JVM) a monthly analysis of job advertisements (which are classified to a detailed occupational code) appearing in a single edition each month of 14 major newspapers and two internet job boards

- a short, large sample. Survey of Employers who have Recently Advertised (SERA Extensive) skilled vacancies to establish in which skilled occupations employers are having difficulty filling vacancies

- an intensive, small sample, largely qualitative survey (SERA Intensive) of employers who have advertised vacancies in selected skilled occupations in shortage, to gain an in-depth understanding of these shortage.

- analysis of statistical sources on supply and demand forces contributing to shortages in selected occupations

- detailed reports on these selected occupations.

\section{Introduction}

The Job Vacancy Monitoring Programme is one of the initiatives of the government's Skills Action Plan (SAP). The SAP is a whole-of-government strategy that aims to speed up the matching of people's skills to the job opportunities that are currently available and reduce skill shortages in the future by helping people to make informed decisions about education and training. In order to improve this matching process we need to identify areas of current skill shortage and to understand the forces that are contributing to these shortages. The JVMP was developed to provide these insights.

The JVMP was designed after a comprehensive process of stakeholder consultation and examination of the international experience with vacancy and skill shortage surveys.

\section{Planning the JVMP: Review of International Experience and Consultation with Stakeholders}

The process of selecting and designing an appropriate approach for the collection of skill shortage information in New Zealand involved a review of international best practices, two rounds of consultations with potential users, the design of four potential survey options and a critical assessment of these options with respect to their applicability to data needs in New Zealand. These steps are summarised below. Further details are contained in a Department of Labour report titled Identification of $a$ survey option for the collection of vacancy information in New Zealand (Department of Labour, 2003).

\section{International Experience with Skill Shortage and Vacancy Surveys}

Thirteen vacancy and skill shortage surveys from around the world were closely examined. They were classified into five broad approaches, which formed the basis for the development of the range of programme options that were considered for implementation in New Zealand. The five categories are:

Model A: A short quarterly, representative survey of employers that gives an aggregate measure of vacancies i.e. the total number of vacancies at a point in time, but does not provide any characteristics of the vacancies.

Model B: A twice yearly representative survey of employers that provides estimates of the number of vacancies in the economy as a whole and the characteristics of these vacancies such as occupation and the required level of education and experience.

Model C: This model is a combination of a quarterly aggregate survey and a detailed annual supplement survey. This supplement typically aims to describe the characteristics of vacancies in terms of occupation, location, level of experience and education needed.

Model D: This model is a considerably expanded version of Model B that is only conducted once a year. It not only measures the number and characteristics of vacancies but it also provides an extensive coverage of a wide number of vacancy and skill shortage issues, or an intensive probing of a limited number of issues. 
Model E: This is an annual survey of employers who have recently advertised vacancies. The coverage of the survey is limited to a number of selected professional and trade occupations, which are suspected to be experiencing shortages. Although it provides some quantitative indicators its emphasis is on qualitative information.

The review of international surveys also highlighted a number of design issues that required consideration when constructing the survey options for New Zealand. The design issues identified include the method of data collection, the use of existing surveys as a vehicle of data collection, the identification of the respondent unit and sectoral coverage.

\section{Data Needs of Potential Users of the Survey}

Following the investigation into the international experience with vacancy surveys, a survey of user needs was conducted among a sample of 32 potential users of the data. The survey investigated the objectives of potential users that skill shortage information could help to achieve, identified skill shortage and vacancy information sources that were currently used and probed the users views on trade-offs in the design of the survey in terms of the depth of coverage, frequency and timeliness.

The main thrust of the survey was to gain an understanding of which issues potential users would like to see covered in the skill shortage survey. Users were presented with a list of 39 potential questions or variables that could be collected and asked to rate them according to their importance. The following conclusions were drawn with regard to the questions and variables:

- The new measure of vacancies or skill shortages should provide detailed titles of occupations that are experiencing shortages rather than broader categorisations.

- A considerable proportion of the survey respondents have indicated that they need an understanding of the demand and supply forces contributing to shortages or surpluses within occupations.

- A regional perspective is necessary.

- The survey needs to be able to identify which vacancies are difficult-to-fill as this provides a pointer to possible skill shortages. However, the survey should distinguish between difficult-to-fill vacancies that arise due to genuine skill shortages and those that arise due to more general recruitment difficulties because of unattractive conditions such as pay or unsociable hours.

- The survey has detected a need for an understanding of some of the skill requirements of vacancies that are difficult-to-fill.

\section{Design of Four Options for Consideration in New Zealand}

Four survey options were constructed, largely on the basis of the international models identified earlier, for consideration in New Zealand. The surveys vary in terms of their frequency, method of data collection, mix of outputs, type of information collected and the representivity of the sample of employers on which they are based.

The size of the sample of employers was given careful consideration. Estimates by LMPG show that considerable sample sizes will be required to provide statistically reliable estimates of vacancies to third and fourth digit occupational level. Even a sample size of 50,000 establishments will only provide publishable vacancy estimates for two-thirds of four-digit occupations. A sample size of 80,000 will take this proportion up to $90 \%$. However, the budget allocation to the vacancy survey does not permit samples of these sizes.

\section{Option One: Quarterly short with detailed annual supplement}

This option combines a short quarterly representative survey (quarterly sample size $=7,500$ ) that measures the total number of vacancies with an annual supplement ( sample $=20,000$ ) that collects information about the characteristics of vacancies in terms of occupation, location, level of experience, education needed, remuneration offered and the duration of the vacancy.

\section{Option Two: Annual detailed occupation}

This representative survey is similar to the annual component of option one. The resources freed up from dropping the quarterly component are used to increase the sample size $(35,000)$ with the view to delivering the maximum possible occupational detail within the constraints of the budget. The questionnaire is short with a limited number of closed questions.

\section{Option Three: Annual telephonic}

This representative survey (sample $=20,000$ ) goes beyond merely measuring the number and characteristics of vacancies and collects limited qualitative information such as the reasons for the vacancy being difficult to fill and the skills that are difficult to obtain from applicants to difficult-to-fill vacancies. The questionnaire is considerably longer and more complicated than the previous option.

\section{Option Four: Survey of emplovers that have recently advertised (SERA) and associated activities}

This option differs from the previous three, as it is not based on a representative survey of employers. It comprises two surveys of employers that have recently advertised job vacancies as well as a number of complimentary non-survey activities. As this option was finally chosen as the selected option it is described in more detail in the final section of the executive summary.

\section{User Views of Four Options}

A second round of consultations with potential users was conducted to investigate user preferences with regard to the four selected survey options. The majority of 
respondents $(71 \%)$ identified the fourth option as their preference. The major reasons mentioned by respondents was the richness of the qualitative information around occupations that were considered to be in shortage. Other reasons included its more focussed approach, ability to distinguish between recruitment difficulties and skill shortages, cost effectiveness, lower respondent burden and useful mix of outputs.

Option one was preferred by $21 \%$ of respondents, mainly because of the frequency of its output. This option would provide users with an insight into where tightness was occurring in the labour market, state of the business cycle, intentions of employers to increase employment and the performance of the labour market.

Option three was identified by $8 \%$ of respondents as their chosen option. Their choice was based on its ability to provide a big picture of the labour market combined with a wide coverage of occupations and collection of qualitative information, which provided some richness to the statistics.

\section{Assessment of Four Options}

The four selected survey options were assessed against a list of twelve criteria with the view to selecting a survey option for further development and implementation in New Zealand. Option four performed the best, or joint best against nine of the twelve criteria. It was estimated that option four could deliver the majority of user needs (criterion 1) as determined in the first round of user consultations and was the preference of users (2) as measured in the second round of user consultations. It again performed best when the strategic importance (3) of potential users was considered. Option four was able to deliver information that made a clear contribution to the objectives of SAP (4) and its output did not duplicate existing or forthcoming information (5). It was the most cost-effective (6) and imposed the lowest respondent burden (7) of the four options. Furthermore it had the greatest ability to focus on specialised occupations (8) and has the potential to create strong linkages with other initiatives (11) concerned with skill shortage issues. Option four performed poorly against the criteria of statistical robustness (9), ability to provide a macroperspective (10), and objectivity (12).

\section{Final Decision on Programme Option}

On the basis of the assessment of the four programme options outlined above the Steering Group agreed that option four should be implemented. However, it was noted that the cost of option four is likely to be less than the allocated budget. For this reason it was decided to further investigate the possibility of supplementing option four with a short quarterly survey for measuring the aggregate number of vacancies in the economy. This survey could provide a macro-perspective of skill shortages and thereby overcome an important shortcoming of option four. Further clarification of costings, top-up funding, collection methods and its contribution to broader labour market statistics needs to be obtained.
The chosen programme option differs from the other three, as it is not based on a representative survey of employers. It comprises two surveys of employers that have recently advertised job vacancies as well as a number of complimentary non-survey activities. The survey components focus on employers that have recently advertised job vacancies as these employers are well positioned to comment on skill shortages.

\section{Structure of JVMP}

This section provides greater detail of the components of the programme that was chosen through the process of stakeholder consultation and review of international practises, as outlined in the above section. The various components of the programme are discussed separately.

\section{Job Vacancy Monitor}

The Job Vacancy Monitor (JVM) is a monthly analysis of job advertisements in selected editions of 14 major newspapers around New Zealand and 2 internet job boards. The purpose of the job ad series is to monitor changes in occupational labour markets. It also provides the basis of the sample for the Survey of Employers who have Recently Advertised (SERA).

Each month all advertisements appearing in a single edition (first Saturday of the month) of 14 major newspapers around the country are captured electronically. A number of fields of information from each advertisement are captured including job title, location and number of vacancies. The job title for each advertised vacancy is coded to a 5-digit occupation using the New Zealand Standard Classification. Likewise location of the vacancy is coded to a standard classification of Urban Areas and Region. Advertisements that are repeated across newspapers are identified and discarded. In addition to monitoring newspaper advertisements the JVM also monitors two Information Technology Internet job boards. The number of new vacancies posted over the previous seven days is counted each Friday.

The results of the JVM are published in a monthly report which is posted on the Department of Labour website (http://www.dol.govt.nz/publications/jvm).

\section{Survey of Employers who have Recently Advertised - Extensive}

The Survey of Employers who have Recently Advertised (SERA) - Extensive is a short telephonic survey of employers who have advertised professional, technical/semi-professional or trade vacancies. The purpose of the survey is to identify skilled occupations in which employers are having difficulty filling vacancies. It provides a broad overview of the skilled labour market.

The survey is conducted between February and May each year. Its sample is drawn from the January, February and March editions of the JVM as well as two additional newspaper editions in mid January and mid-February. 
The sample will contain between 3,000 to 4,000 advertised vacancies. Employers are approached six to eight weeks after advertising and asked whether they have filled their vacancy and how many suitable applicants applied. From this information we are able to calculate a fill rate for each occupation. The fill rate describes the proportion of vacancies that were filled within six to eight weeks of advertising. We are also able to calculate the average number of suitable applicants per vacancy. These measures are key indicators of shortage. Occupations with fill rates lower than about $80 \%$ are generally regarded as being in shortage.

Summary results of the Extensive survey will be published in the middle of the year.

\section{Identification of Key Occupations}

The next step of the JVMP process is to identify key occupations for further in-depth analysis. A number of criteria are used to select key occupations. The first is that there is some evidence that the occupation is in shortage. Evidence of shortage may be drawn from the SERA Extensive, JVM (a rapid increase in the number of advertised vacancies may indicate that a shortage is emerging) or from anecdotal sources if the occupation is not adequately covered in SERA Extensive. Key occupations may also be selected on the basis that they are of considerable strategic importance to the New Zealand economy and do not necessarily have to be in shortage all the time to be included.

\section{SERA Intensive}

This is a survey of employers who have recently advertised vacancies in the key occupations identified in the previous step. Its objective is to gain in-depth knowledge of the causes of shortages in each occupation. The survey is conducted in the second half of the year and it draws its sample from the Job Vacancy Monitor. The sample may be complemented from other sources of advertising such as professional and trade journals and internet job boards. The survey is based on a sample of 20 employers for each occupation.

The questionnaire is a mix of quantitative and qualitative questions. Similar quantitative questions to those used in the Extensive are included to enable a fill rate and average number of suitable applicants per vacancy to be calculated for each occupation. Additional qualitative questions cover the following issues:

- The supply and demand forces that are contributing to shortages

- Whether the shortage is a genuine skill shortage or a recruitment difficulty

- Implication for the employer and their response to their inability to fill vacancies

- Skill sets that employers are unable to obtain in applicants to their vacancies.

Sixteen trade and eleven professional occupations were included in the 2004 SERA Intensive.

\section{Quantitative Research}

Various data sources that provide additional insight into the supply and demand forces contributing to shortages in each of the key occupations are analysed. These sources include:

- Enrolments and completions for courses related to the occupation;

- Migration of persons qualified in the occupation;

- Employment data from sources such as Household Labour Force Survey, censuses and employer bodies;

- Economic data related to the economic sector in which the occupation is concentrated.

\section{Occupational Reports}

Comprehensive reports on each of the key occupations will be published. These reports tie together information gathered from the SERA Intensive and analysis on other statistical sources. The reports will include the following:

- Trends in demand and supply based on analysis of statistical sources and employer insights.

- Employer recruitment experiences i.e. ability of employers to fill their vacancies

- A labour market rating. A judgement will be made whether the occupation is in shortage, is characterised by recruitment difficulties or there is no shortage.

- Short term outlook. A judgement will be made whether the current labour market situation will persist or is likely to change over the next 12 months.

Draft reports will be distributed to industry and occupational bodies such as industry training organisations and professional associations. Feedback on analysis and conclusions will be incorporated into final reports.

\section{Conclusion}

The Job Vacancy Monitoring Programme was implemented to provide new insights into the depth and extent of skill shortages in New Zealand and to provide an understanding of the forces contributing to shortages. It will provide new information which is grounded in sound research enabling government make better decisions around training and education and the importation of skills into New Zealand to alleviate shortages.

\section{References}

Department of Labour (2003). Identification of a Survey Option for the Collection of Vacancy Information in New Zealand 